\title{
Pseudopotential for the electron-electron interaction
}

\author{
J.H. Lloyd-Williams, ${ }^{1}$ R.J. Needs, ${ }^{1}$ and G.J. Conduit ${ }^{1}$ \\ ${ }^{1}$ Cavendish Laboratory, J.J. Thomson Avenue, Cambridge, CB3 OHE, United Kingdom
}

(Dated: July 16, 2015)

\begin{abstract}
We propose a pseudopotential for the electron-electron Coulomb interaction to improve the efficiency of many-body electronic structure calculations. The pseudopotential accurately replicates the scattering properties of the Coulomb interaction, and recovers the analytical solution for two electrons in a parabolic trap. A case study for the homogeneous electron gas using the diffusion Monte Carlo and configuration interaction methods recovers highly accurate values for the ground state energy, and the smoother potential reduces the computational cost by a factor of $\sim 30$. Finally, we demonstrate the use of the pseudopotential to study isolated lithium and beryllium atoms.
\end{abstract}

PACS numbers: 71.15.Dx, 31.15.A-

Electron-electron interactions drive chemical reactions, govern material properties, and conspire to form strongly correlated phases. Despite the widespread and important consequences of electronic correlations, leading computational techniques such as diffusion Monte Carlo (DMC) [1], truncated configuration interaction (CI) [2, 3], Møller-Plesset theory [4], coupled cluster theory [5], and F12 methods [6]. These approaches are very expensive for real-life systems because the divergence in the electron-electron Coulomb interaction must be sampled finely $[7,8]$. Here we propose a pseudopotential that accurately replicates the scattering properties of the Coulomb interaction, delivers the ground state energies within chemical accuracy of $1 \mathrm{kcal} \mathrm{mol}^{-1}$, but does not diverge, which reduces the computational cost of both $\mathrm{DMC}$ and CI by a factor of $\sim 30$.

Pseudopotentials were first introduced by Hellmann [9] to describe the attractive electron-ion interaction in molecules and solids. Integrating out the core electrons that screen the central ion leaves a pseudopotential for the valence electrons. The reduction in the number of electrons and the greater smoothness of the electron-ion pseudopotential provides computational advantages that led to their widespread adoption in electronic structure calculations, including density functional theory [10] and DMC methods [11].

First principles approaches must still account for the divergent repulsive electron-electron interaction that necessitates fine sampling $[7,8]$. The Kato cusp conditions [12-19] enforce a wavefunction with a kinetic energy divergence that cancels the Coulomb divergence, leaving a remnant finite discontinuity in the local energy $\psi^{-1}(\mathbf{R}) \hat{H} \psi(\mathbf{R})$, which is evaluated with the electrons at point $\mathbf{R}$ in configuration space. There have been attempts to apply a local density solution to the shortranged behavior $[20,21]$. It was also proposed to introduce a soft-Coulomb operator either in real space [22], or reciprocal space [23]. Another attempt was to split the Coulomb interaction into a short and long-ranged component, so that they could be handled separately [24]. However, at present pseudopotentials are not generally used to smooth the electron-electron interaction.

We develop an accurate electron-electron pseudopotential for electrons scattering with any energy and angular momentum. We build on the formalism used to construct a pseudopotential for the contact interaction found in ultracold atomic gases [25]. This formalism is somewhat different from the standard pseudopotential approach developed for attractive electron-ion interactions that focuses on discrete bound state energies [26-29], although it can be extended to scattering states [30]. The proposed pseudopotential is identical to the Coulomb interaction outside of a cut-off radius where many-body physics becomes important. The pseudopotential delivers all of the physics of the Coulomb interaction but does not diverge, so that the ground state can be determined efficiently. After developing the pseudopotential in the two-body scattering problem, we test it on the analytically solvable system of two electrons in a parabolic trap [31].

We study the applicability, accuracy, and portability of the pseudopotential for a homogeneous electron gas (HEG) using two methods: DMC in which the use of the pseudopotential reduces the required time-step, and CI in which the pseudopotential reduces the size of the plane-wave basis set required. The pseudopotential delivers chemical accuracy, and at the same time reduces the computational cost of both techniques by a factor of $\sim 30$. Finally, we test the pseudopotential on two inhomogeneous systems, the isolated lithium and beryllium atoms.

\section{CONSTRUCTION OF THE PSEUDOPOTENTIAL}

To construct the pseudopotential, we adopt the formalism of Ref. [25] and study the two-body problem: two electrons in their center-of-mass frame with wave vector $k \geq 0$ and angular momentum quantum number $\ell$. The Hamiltonian in atomic units is $-\frac{1}{r^{2}} \frac{\mathrm{d}}{\mathrm{d} r}\left(r^{2} \frac{\mathrm{d} \psi}{\mathrm{d} r}\right)+\frac{\ell(\ell+1)}{r^{2}} \psi+$ $V(r) \psi=k^{2} \psi$, and the repulsive Coulomb potential is $V(r)=1 / r$. The proposed pseudopotential is identical to the Coulomb potential outside of a cutoff radius $c$, 
(a) Pseudopotential

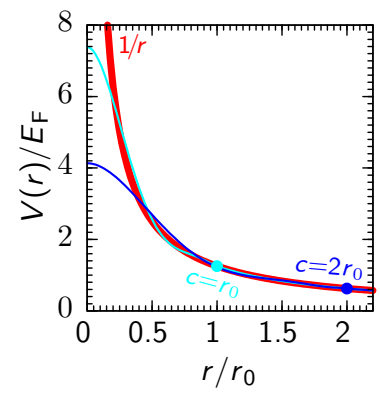

(c) Error with density

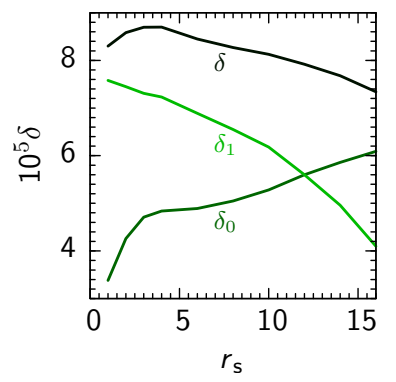

(b) Error with cutoff

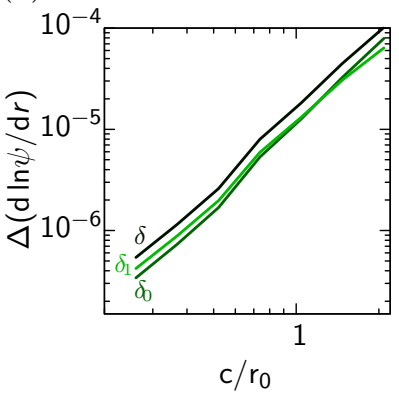

(d) Two-body scattering

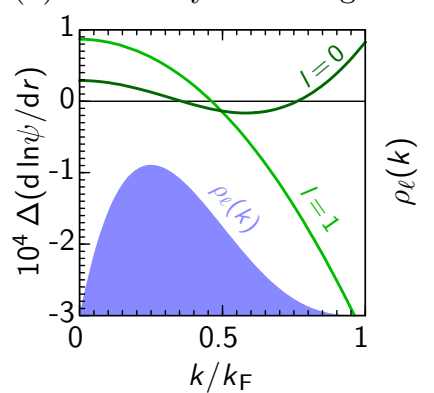

FIG. 1. (Color online) (a) The interaction potentials: the Coulomb potential is shown in red, the pseudopotential with cutoff radius $c=r_{0}$ is in cyan, and the pseudopotential with cutoff radius $c=2 r_{0}$ is in blue. (b) The error in the logarithmic derivative of the scattering wavefunction with cutoff radius for an electron gas with $r_{\mathrm{s}}=2 . \quad \delta$ shows the error summed over angular momentum channels, $\delta_{0}$ is the contribution from the $\ell=0$ channel and $\delta_{1}$ from the $\ell=1$ channel. (c) The pseudopotential error for a range of $r_{\mathrm{s}}$ values. (d) The error in the logarithmic derivative of the scattering wavefunction with incident wave vector for the $\ell=0$ and $\ell=1$ scattering channels. The filled blue curve plotted on an arbitrary linear scale on the secondary y-axis shows the weighting factor $\rho_{\ell}(k)$ used in evaluating the overall error in the logarithmic derivative.

and at the cutoff it is continuous and has a continuous first derivative. At small electron-electron separation $r$, the pseudopotential can be chosen to be softer than the Coulomb interaction so that on electron coalescence at $r=0$ it is finite and has zero gradient to remove possible divergences and discontinuities in the local energy, thereby reducing the variance in our estimate of the total energy. These considerations suggest a pseudopotential of the form

$$
V(r)=\frac{1}{c} \begin{cases}1+\left(1-\frac{r}{c}\right)\left(\frac{r}{c}\right)^{2}+ & \\ \left(1-\frac{r}{c}\right)^{2}\left[v_{1}\left(\frac{1}{2}+\frac{r}{c}\right)+\sum_{i=2}^{N_{\mathrm{v}}} v_{i}\left(\frac{r}{c}\right)^{i}\right] & 0 \leq r \leq c \\ \frac{c}{r} & r>c,\end{cases}
$$

with variational freedom introduced by a polynomial expansion of order $N_{\mathrm{v}}=6$. To determine the parameters $\left\{v_{i}\right\}$ we calculate the scattering states. The scat-

tering states $\psi_{k, \ell}(r)$ for the Coulomb interaction can be solved exactly in terms of Whittaker functions, whereas the scattering solution $\phi_{k, \ell}(r)$ from the pseudopotential is solved numerically. The difference between the scattering properties of the two potentials is characterized by the mean square error in the logarithmic derivative of the scattering wavefunction at the cutoff radius

$$
\delta^{2}=\sum_{\ell=0}^{6} \int_{0}^{k_{\mathrm{F}}} \mathrm{d} \mathbf{k} \rho_{\ell}(\mathbf{k})\left(\left.\frac{\mathrm{d} \ln \psi_{k, \ell}}{\mathrm{d}(r / c)}\right|_{r=c}-\left.\frac{\mathrm{d} \ln \phi_{k, \ell}}{\mathrm{d}(r / c)}\right|_{r=c}\right)^{2},
$$

which is summed over all angular momentum channels $\ell=\{0, \ldots, 6\}$ and integrated over all possible scattering wave vectors $0 \leq k \leq k_{\mathrm{F}}$ encountered in an electron gas with Fermi momentum $k_{\mathrm{F}}$ [29]. Following Ref. [25], we weight the importance of different scattering states by a factor $\rho_{\ell}(k)$, which is chosen to replicate the density of scattering states in a Hartree-Fock trial wavefunction for a homogeneous electron gas where $\rho_{\ell}(\mathbf{k})=\int \mathrm{d} \mathbf{q} n_{\mathrm{F}}(\mathbf{q}) n_{\mathrm{F}}(\mathbf{k}+\mathbf{q}) / \sqrt{(2 \ell+1) ! !}$, and $n_{\mathrm{F}}$ is the Fermi-Dirac distribution function. We select the variational parameters $\left\{v_{i}\right\}$ that minimize $\delta^{2}$, which gives a pseudopotential whose scattering closely replicates the Coulomb interaction. We associate the length scale $r_{0}=(9 \pi / 4)^{1 / 3} / k_{\mathrm{F}}$ with the typical electron separation and characterize an electron gas with the standard density parameter $r_{\mathrm{s}}=r_{0} / a_{\mathrm{B}}$, where $a_{\mathrm{B}}$ is the electron Bohr radius)

In Fig. 1(a) we examine two of the pseudopotentials constructed to be used in an electron gas with density $r_{\mathrm{s}}=2$. At small $r$ the pseudopotential is flat to ensure that the wavefunction is smooth. The pseudopotential is therefore weaker than the Coulomb potential but, to give the same net scattering strength, the pseudopotential must exceed the Coulomb potential at intermediate $r$, before they merge at the cutoff radius. The figure also shows that on reducing the cutoff radius the pseudopotential approaches the Coulomb potential. Therefore, the pseudopotential should recover the scattering properties of the Coulomb potential with increasing accuracy as the pseudopotential cutoff radius is reduced. We verify this in Fig. 1(b) where the error in the logarithmic derivative of the scattering wavefunction falls with cutoff radius as $\sim\left(c / r_{0}\right)^{2.6}$. The $\ell=0$ and $\ell=1$ channels provide similar contributions to the error in the scattering wave function. Now that we have tested the pseudopotential developed for an electron gas at $r_{\mathrm{s}}=2$, we develop and test pseudopotentials to be applied to electron gases with the full range of densities $1 \leq r_{\mathrm{s}} \leq 16$ that can be found in real-life systems. Fig. 1(c) shows that the average error in the logarithmic derivative $\delta \lesssim 10^{-4}$ is small compared with the typical scattering phase shift $2 \pi$ over a wide range of electron gas densities, demonstrating that the pseudopotential accurately reproduces the two-body scattering properties of the Coulomb interaction.

The error in the logarithmic derivative of the wavefunc- 
(a) $\ell=0$ wavefunction

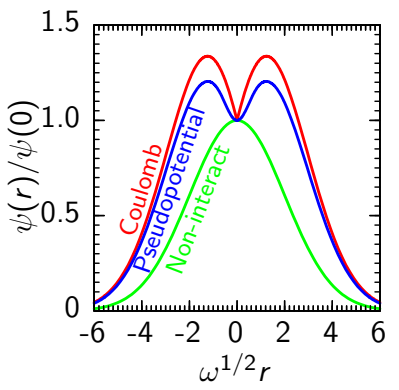

(b) Two-body scattering

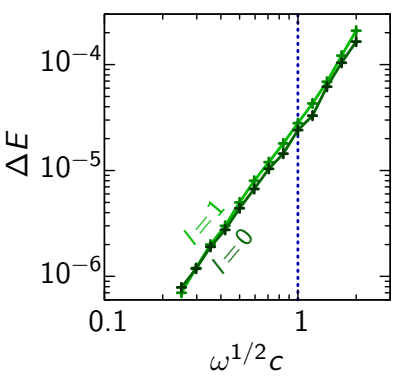

FIG. 2. (Color online) (a) Wavefunction of the relative motion of two electrons in a harmonic trap in the $\ell=0$ angular momentum state. The green curve shows the wavefunction for non-interacting electrons, the red shows electrons interacting via the Coulomb interaction, and blue shows interactions via the pseudopotential. (b) The error per electron in the estimate of the ground state energy of two opposite-spin $(\ell=0)$ and same-spin $(\ell=1)$ electrons in a parabolic trap as a function of cutoff radius. The vertical blue dotted line shows the typical separation of the electrons in the harmonic trap.

tion averaged over the incident wave vectors of electrons scattering off the pseudopotential is small. To understand how this is achieved, we examine in Fig. 1(d) the variation of the error in the logarithmic derivative with respect to the incident wave vector. The $\ell=0$ channel has a quadratic form that crosses zero error twice, whereas the $\ell=1$ channel has an error that crosses zero only once. The variational freedom in the pseudopotential has been used to minimize the error around $k \approx 0.3 k_{\mathrm{F}}$ where the density of scattering states is largest, sacrificing accuracy at higher incident wave vectors.

With the pseudopotential providing phase shifts with an error of only $\sim 10^{-4}$, we are well-positioned to test its performance in a many-body setting. We first study an idealized system with an analytical solution to provide an exact benchmark: two electrons in a parabolic trap. We also study systems that cannot be solved analytically: the HEG with two complementary methods; DMC and CI; and we also study isolated lithium and beryllium atoms. This allows us to assess the performance and accuracy of the pseudopotential, and verify its portability.

\section{TWO ELECTRONS IN A PARABOLIC TRAP}

Now that we have constructed the Coulomb pseudopotential and calibrated it against the phase shift of two atoms scattering in a vacuum, we evaluate the accuracy of the pseudopotential in a second analytically soluble system: Hooke's atom, two interacting electrons trapped in the parabolic well $m \omega^{2} r^{2} / 2$ [31]. This problem received early numerical attention [32-34], and was more recently studied with coupled cluster methods [35, 36].
We solve separately for opposite- and same-spin electrons as the relative wavefunctions differ due to fermion antisymmetry.

We solve for the energy of two interacting electrons in the parabolic trap within the center-of-mass frame in which the interacting Hamiltonian for relative motion is $\hat{H}=-\frac{1}{r^{2}} \frac{\mathrm{d}}{\mathrm{d} r}\left(r^{2} \frac{\mathrm{d}}{\mathrm{d} r}\right)+\omega^{2} r^{2} / 4+\ell(\ell+1) / r^{2}+V(r)$, where $V(r)=1 / r$ is the Coulomb interaction in atomic units. For the special case of $\omega=1 / 8$ this model can be solved analytically for the $\ell=0$ (opposite-spin electrons) ground state giving eigenenergy $E=5 / 4$ (the noninteracting center-of-mass Hamiltonian has energy $3 / 4$ giving a total energy $E=2$ ). On replacing the interaction potential by a pseudopotential, the Hamiltonian for relative motion can be solved numerically and the ground state energy compared with the exact solution for the Coulomb interaction. When constructing the pseudopotential we chose a maximum energy of the scattering states that we integrate over in Eqn. (1). We take this to be the energy per electron in the interacting system, $E=1$.

The parabolic trap is an ideal setting to compare the ground state wavefunction predicted by the Coulomb interaction with that from the pseudopotential. In Fig. 2(a) we show the $\ell=0$ (i.e., opposite-spin electrons) ground state wavefunction for relative electron motion. Firstly, to orient the discussion we show the wavefunction for non-interacting electrons, which is a Gaussian that is smooth at electron coalescence. The wavefunction for the Coulomb interaction has a gradient discontinuity at electron coalescence which provides a divergent kinetic energy that cancels the divergence in the Coulomb interaction. In general the gradient discontinuity is difficult to capture numerically and it hinders computational approaches. However, the smooth pseudopotential provides a wavefunction that is smooth over all space including at electron coalescence, which should aid computational methods.

In Fig. 2(b) we study the error in the $\ell=0$ ground state energy when varying the cutoff radius, which is the control parameter for adjusting the accuracy of the pseudopotential. The error in the ground state energy with the cutoff set to the typical electron separation, $1 / \sqrt{\omega}$, is $2 \times 10^{-5}$ au per electron. With decreasing cutoff radius $c$ the pseudopotential approaches the Coulomb interaction and the accuracy further increases, varying as $\sim(\sqrt{\omega} c)^{2.7}$. This scaling in error with cutoff radius is similar to that seen in the error in the logarithmic derivative of the scattering wavefunction shown in Fig. 1(b), which varies as $\sim\left(c / r_{0}\right)^{2.6}$.

The interactions between opposite-spin and same-spin electrons both make important contributions to the total energy in many systems. Therefore, we next study the ground state energy of same-spin electrons in a parabolic trap. This requires a spatially anti-symmetric ground state, and so we require the system with $\ell=1$. Here 
the system is analytically soluble with $\omega=1 / 16$ giving an energy of $E=21 / 16$. In Fig. 2(b) we study the error in the prediction of the $\ell=1$ energy. The error in the ground state energy with the cutoff set to the typical electron separation, $1 / \sqrt{\omega}$, is $3 \times 10^{-5}$ au per electron. With decreasing cutoff radius $c$ the accuracy further increases, varying as $\sim(\sqrt{\omega} c)^{2.55}$. The errors achieved for both the $\ell=0$ and $\ell=1$ channels are two orders of magnitude better than the target chemical accuracy of $1 \mathrm{kcal} \mathrm{mol}^{-1}=0.0016 \mathrm{au}$ per electron. The proposed pseudopotential is therefore sufficiently accurate for scattering between both opposite- and same-spin electrons in this two-body system.

\section{HEG WITH DIFFUSION MONTE CARLO}

The pseudopotential was calibrated using the exactly soluble two-body scattering problem and tested against the analytic solution of two electrons in a parabolic trap. We now study a system that cannot be solved analytically: the HEG. We must rely on a numerical approach to determine the ground state energy, allowing us to expose the computational benefits of using a pseudopotential. We first study the HEG with DMC as this is the leading approach for accurate calculations of the ground state energy [38-41].

We have used the CASINO quantum Monte Carlo code [42] to perform variational and diffusion Monte Carlo (VMC and DMC) calculations [1, 38]. The Metropolis algorithm is used in the VMC method to generate a set of electron configurations distributed according to the square modulus of the trial wavefunction over which the local energy is averaged. In the DMC method, an initial wavefunction is evolved in imaginary time, which projects out the ground state. The antisymmetry of the wavefunction is imposed via the variational fixed-node approximation, in which the nodal surface remains unchanged during the evolution. The simulation proceeds with configurations undergoing drift, diffusion and birth/death processes, which simulate the evolution of the wavefunction in imaginary time. DMC provides an upper bound on the energy that is lower than the VMC bound calculated with the same trial state.

We focus on a three-dimensional homogeneous electron gas with $N_{\uparrow}=N_{\downarrow}=57$ electrons and density $r_{\mathrm{s}}=r_{0} / a_{\mathrm{B}}=2$ with $a_{\mathrm{B}}$ the electron Bohr radius. The calculation is performed in a periodically repeated simulation cell and the interaction energy is calculated using Ewald summation [43, 44]. We first construct a variational wavefunction $\psi=\mathrm{e}^{J} D$ that is the product of a Jastrow factor $\mathrm{e}^{J}$ and a Slater determinant $D=\hat{\mathcal{A}}\left\{\prod_{i \in N_{\uparrow}}^{\mathbf{k} \in k_{\mathrm{F}}} \mathrm{e}^{\mathrm{i} \mathbf{k} \cdot \mathbf{r}_{i}}\right\} \hat{\mathcal{A}}\left\{\prod_{i \in N_{\downarrow}}^{\mathbf{k} \in k_{\mathrm{F}}} \mathrm{e}^{\mathrm{i} \mathbf{k} \cdot \mathbf{r}_{i}}\right\}$, where $\hat{\mathcal{A}}$ is the anti-symmetrization operator that accounts for fermion statistics. The lowest energy plane-wave states $\mathbf{k}$ are used to form the orbitals and periodic boundary conditions are

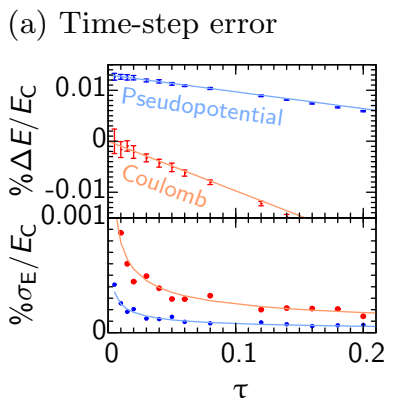

(b) Error with cutoff radius

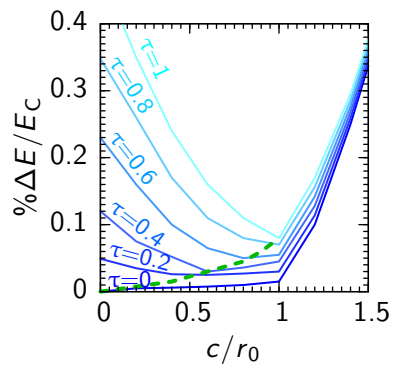

(c) Uncertainty with cutoff

(d) Pair correlation function
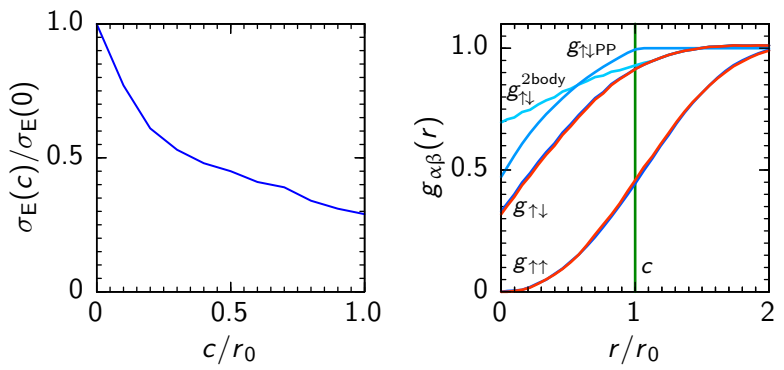

(e) Error with density

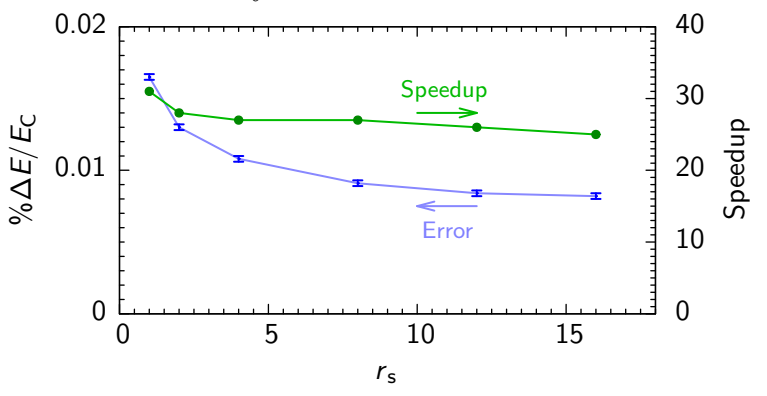

FIG. 3. (Color online) (a) Upper: The error in the energy of the HEG with DMC time step. The y-axis origin is set by the extrapolation to zero time step of the energy obtained with the Coulomb interaction. The red error bars show the Coulomb interaction and the blue error bars the pseudopotential, the solid lines show linear extrapolation to zero time step. Lower: The uncertainty in energy predictions, with the lines showing the $\tau^{-1 / 2}$ fit. (b) The error in the energy with cutoff radius for different time steps $\tau \in\{0, \ldots, 1\}$. Each curve has a minimum with cutoff radius, the locus of these minima with varying time step is tracked by the green dashed line. (c) The relative statistical uncertainty with cutoff radius in DMC. (d) The spin-resolved pair correlation functions for same-spin $\left(g_{\uparrow \uparrow}\right)$ and opposite-spin $\left(g_{\uparrow \downarrow}\right)$ electrons for the Coulomb interaction are shown in red and those for the pseudopotential are in blue. The dotted blue curve shows the analytic correction $\left(g_{\uparrow \downarrow}^{2 \mathrm{body}}\right)$ applied to the cyan $g_{\uparrow \downarrow \mathrm{PP}}$ obtained directly from the pseudopotential. (e) The error in the energy and speedup obtained with density.

applied. The log of the Jastrow factor is

$$
J=\sum_{\substack{\alpha, \beta \in\{\uparrow, \downarrow\} \\ i, j \in N_{\alpha}, N_{\beta}}}\left[1-\frac{\left|\mathbf{r}_{i}-\mathbf{r}_{j}\right|}{L_{\mathrm{u}}}\right]^{3} \Theta\left(L_{\mathrm{u}}-\left|\mathbf{r}_{i}-\mathbf{r}_{j}\right|\right) \sum_{k=0}^{N_{\mathrm{u}}} u_{k \alpha \beta}\left[\frac{\left|\mathbf{r}_{i}-\mathbf{r}_{j}\right|}{L_{\mathrm{u}}}\right]^{k}
$$


which includes strongly repulsive electron-electron correlations. We describe $J$ by a polynomial expansion of order $N_{\mathrm{u}}=8$ in the electron-electron separation, [15] and $L_{\mathrm{u}}$ is a cutoff length. The behavior of the Jastrow factor at electron coalescence can be fixed by the Kato cusp conditions [12]; for the Coulomb potential we can remove the cusp by setting $u_{1 \alpha \beta}=3 u_{0 \alpha \beta}+1 / 2$ for antiparallel spins $(\alpha \neq \beta)$ and $u_{1 \alpha \alpha}=3 u_{0 \alpha \alpha}+1 / 4$ for parallel spins. However, this scheme leaves a remnant discontinuity in the local energy. On the other hand, the pseudopotential is smooth at $r=0$, so there we set $u_{1 \alpha \beta}=3 u_{0 \alpha \beta}$ to ensure that the wavefunction is smooth at electron coalescence. The higher order terms in the Jastrow factor $\left\{u_{i \geq 2, \alpha \beta}\right\}$ provide the freedom to account for longer-ranged correlations. We also add backflow correlations in the Slater determinants using the substitution $\mathbf{r}_{i} \mapsto \mathbf{r}_{i}+\sum_{j \in\left\{N_{\uparrow}, N_{\downarrow}\right\}} \eta_{i j}\left(\left|\mathbf{r}_{i}-\mathbf{r}_{j}\right|\right)\left(\mathbf{r}_{i}-\mathbf{r}_{j}\right)$ with $\eta(r)=\left(1-r / L_{\eta}\right)^{3} \Theta\left(L_{\eta}-r\right) \sum_{k=0}^{N_{\eta}} c_{k} r^{k}$, where $L_{\eta}$ is a cutoff length, and the expansion in variational parameters $c_{k}$ is up to order $N_{\eta}=8$ [45]. The variational coefficients $\left\{u_{k \alpha \beta}, c_{k}, L_{\mathrm{u}}, L_{\eta}\right\}$ are optimized using VMC $[46,47]$.

The VMC wavefunction was used as the trial state for the DMC calculation. DMC propagates the electrons in time step increments $\tau$ governed by Schrödinger's equation in imaginary time. The evolution with a Coulomb interaction must have a small time step to properly sample the rapidly changing local energy near the electron cusp [42]. All DMC calculations were performed with at least 1000 walkers. We use the percentage of the correlation energy $E_{\mathrm{C}}$ retrieved as the measure of the accuracy. There are two main sources of error, firstly the underlying VMC trial wavefunction is not exact, having a variance in the local energy $\sigma_{\mathrm{L}}^{2}=\operatorname{var}\left(\psi^{-1} \hat{H} \psi\right)$ that introduces a systematic error in the DMC estimate of the ground state energy of $\Delta E=a \sigma_{\mathrm{L}} \tau$ [48], where $a$ is a system dependent constant. Secondly, because DMC follows a random walk there is a statistical uncertainty $\sigma_{\mathrm{E}}=b \sigma_{\mathrm{L}} /\left(\tau^{1 / 2} N^{1 / 2}\right)$, where $b$ is a system dependent constant, that can be reduced by taking more samples $N$. Both sources of error increase with the variance of the local energy [49], which for the Coulomb potential is $\sigma_{\mathrm{L}}=7.6 \times 10^{-4} E_{\mathrm{C}}$, and for the pseudopotential (with $c=r_{0}$ ) is $\sigma_{\mathrm{L}}=2.4 \times 10^{-4} E_{\mathrm{C}}$. Using the pseudopotential has resulted in a drop in $\sigma_{\mathrm{L}}$ by a factor of 3.2, which should reduce both the systematic and statistical errors. To expose this we now vary another parameter that enters both sources of error: the time step.

In the upper panel of Fig. 3(a) we first examine the systematic error in the energy. The extrapolates of the ground state energy to zero time step for the Coulomb and pseudopotential interactions agree to within $0.013 \% E_{\mathrm{C}}=0.0012 \mathrm{au}$ per electron [49]. This is better than our goal of chemical accuracy of $1 \mathrm{kcal} \mathrm{mol}^{-1}=0.0016 \mathrm{au}$ per electron. Calculation with the Coulomb interaction and pseudopotential both have the expected $\Delta E=a \sigma_{\mathrm{L}} \tau$ linear variation of energy with time step, though the slope for the Coulomb interaction is 3.5-times as steep as for the pseudopotential interaction. This is consistent with the Coulomb interaction having a $\sigma_{\mathrm{L}}$ that is 3.2-times as large. Now that we have confirmed the analytical form for the systematic error in the energy, we examine the statistical uncertainty that is expected to be $\sigma_{\mathrm{E}}=b \sigma_{\mathrm{L}} /\left(\tau^{1 / 2} N^{1 / 2}\right)$. The lower panel of Fig. 3(a) confirms that the statistical error is well-fitted by a $\tau^{-1 / 2}$ power law, and that the ratio of the fitting coefficients is 3.3 , consistent with the expected ratio from the local energy of 3.2 .

With the behavior of both the systematic and statistical errors verified, we determine the acceleration offered by the pseudopotential. Considering only the statistical error, $\sigma_{\mathrm{E}}=b \sigma_{\mathrm{L}} /\left(\tau^{1 / 2} N^{1 / 2}\right)$, to achieve a target final uncertainty requires a computational effort that scales with the number of samples as $N \sim \sigma_{\mathrm{L}}^{2}$. The local energy calculated with the pseudopotential has an error of $\sigma_{\mathrm{L}}$, which is 3.2-times smaller than for the Coulomb interaction, resulting in a 10-times speedup. However, when using the pseudopotential the systematic error is also reduced, allowing the calculation to be performed at larger time steps, which will also reduce the statistical error as $\sim \tau^{-1 / 2}$. We consider these effects on an even footing by combining the systematic and statistical errors in quadrature to give a total expected error $\Delta E_{\text {tot }}$ in the estimate of the energy of

$$
\begin{aligned}
\Delta E_{\mathrm{tot}}^{2} & =\Delta E^{2}+\sigma_{\mathrm{E}}^{2} \\
& =\sigma_{\mathrm{L}}^{2}\left(a^{2} \tau^{2}+\frac{b^{2}}{\tau N}\right) .
\end{aligned}
$$

The systematic contribution to the total error grows with time step while the statistical uncertainty diverges with decreasing time step. The best compromise between the two can be found by minimizing the error with respect to time step $\tau$ to yield

$$
\min \left(\Delta E_{\mathrm{tot}}\right)=\frac{3^{1 / 2} b^{2 / 3}}{2^{1 / 6} a^{1 / 3}} \frac{\sigma_{\mathrm{L}}}{N^{1 / 3}} .
$$

If we aim for a particular target total error the computational effort scales with the number of samples as $N=\sigma_{\mathrm{L}}^{3}$. The pseudopotential reduces $\sigma_{\mathrm{L}}$ by a factor of 3.2 , and therefore the pseudopotential offers a $\sim 30$ fold reduction in computational cost while delivering chemical accuracy.

With the benefits of the pseudopotential established, in Fig. 3(b) we investigate tuning of the pseudopotential cutoff radius. Starting with a small cutoff radius, the energy has a minimal systematic error at small time steps, but the calculation with the Coulomb interaction suffers from a large local energy variance and the error grows rapidly with time step. As the cutoff is increased the variance in the local energy is reduced and the finite time step error falls until it is minimal at $c \approx r_{0}$. 
At large cutoff radii $c \gtrsim r_{0}$ the interaction potential is insufficiently accurate to reproduce the correct ground state energy in the zero-time-step limit. There is now a high probability that three electrons will be found within the cutoff radius, whereas the pseudopotential was calibrated for two-body physics. The error therefore increases rapidly, independently of the time-step adopted. When selecting the cutoff radius one should also consider the impact of the variance in the local energy on the statistical uncertainty in the final result. In Fig. 3(c) we show that with increasing cutoff radius the increasingly smooth pseudopotential leads to a reduction in the relative uncertainty. At $c=r_{0}$ the relative uncertainty has fallen by the same factor of $\sim 3.2$ as shown in Fig. 3(a).

In Fig. 3(d) we study the modification of the pair correlation function arising from the use of the pseudopotential. The same-spin pair correlation function from the Coulomb interaction and the pseudopotential agree within $0.5 \%$. The opposite-spin correlation functions are identical at separations $r \gtrsim c$ where the underlying potentials are identical. At $r \lesssim c$ the pseudopotential is smaller than the Coulomb potential, and therefore the corresponding pair correlation function is larger. However, at small separations two-body physics dominates, and we can separately calculate the pair correlation function by solving the same two-body scattering problem that we used to form the original pseudopotential. This two-body solution can be used to correct the many-body estimate of the pair correlation function for the incorrect two-body effects, bringing it into agreement with the solution for the Coulomb potential to within 1\%. Any further deviation can be ascribed to three- and higher-body physics that occurs for $r \leq c$, which is rare as the electrons are simultaneously Pauli blocked and repelled by the strong Coulomb repulsion.

Having confirmed the utility, robustness, and accuracy of the pseudopotential for the electron gas with $r_{\mathrm{s}}=2$ we study the accuracy of the pseudopotential for electron gases with densities in the range $1 \leq r_{\mathrm{s}} \leq 16$. With the cutoff radius at each density set according to $c=$ $r_{0}=a_{\mathrm{B}} r_{\mathrm{s}}$, we compare the ground state energy from the pseudopotential with that of the Coulomb interaction. In Fig. 3(e) we see that the pseudopotential is able to deliver ground state energies to better than chemical accuracy with a speedup by a factor of $\sim 30$ across a broad range of densities.

\section{HEG WITH CONFIGURATION INTERACTION}

The success of the pseudopotential for studying the HEG with DMC motivates us to consider a second complementary approach to examine the HEG, Configuration Interaction Doubles (CID) [2,3]. We adopt a plane-wave basis for our CID calculations, which offers a robust test of the portability of the pseudopotential. CID theory (a) Coulomb wavefunction

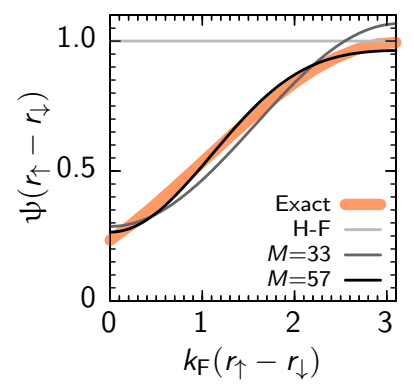

(b) Pseudopot. wavefunction

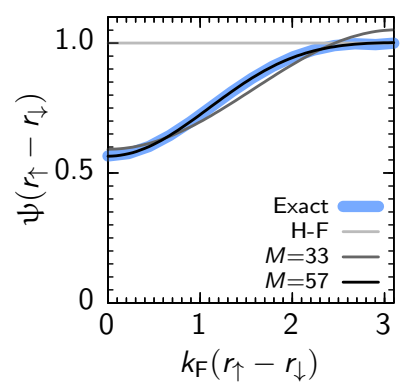

(c) Wavefunction convergence

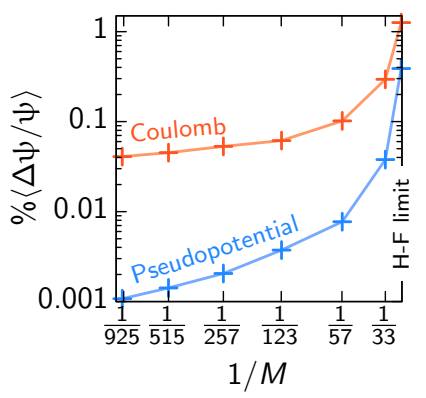

(d) Energy convergence

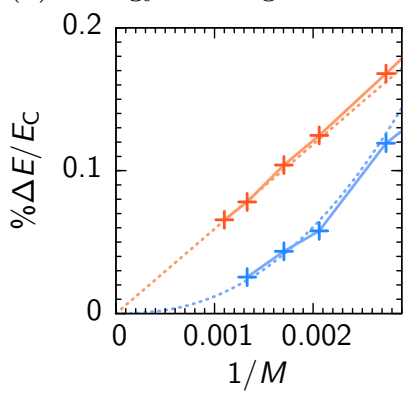

FIG. 4. (Color online) (a,b) The CID wavefunction for opposite-spin electrons passing through coalescence for the HEG at $r_{\mathrm{s}}=2$ with increasing plane-wave orbital basis sets of size $M$. The exact solution is shown in red (blue) for the Coulomb interaction potential and the pseudopotential. (c) The average relative error in the wavefunction, and (d) the percentage error in the energy with basis set size for the Coulomb potential (red) and pseudopotential (blue) with dotted trend lines.

starts from the Hartree-Fock ground state and includes electron correlations through double excitations into the unoccupied (plane-wave) orbitals. In the Coulomb potential, the wavefunction has a gradient discontinuity at electron-electron coalescence that must be described by a large number $M$ of plane-wave basis states with a computational cost that scales as $\mathcal{O}\left(M^{6}\right)$. However, the pseudopotential removes the electron-electron cusp rendering the wavefunction smooth, which therefore should require fewer plane waves to describe the ground state and in turn reduce the computational expense.

The major computational gain offered by the pseudopotential is to aid the description of the behavior at electron coalescence, and therefore we first examine how the wavefunction at coalescence of two opposite-spin electrons evolves with the size of the plane-wave basis set. In the presence of the Coulomb interaction we compare the exact relative wavefunction with that from a finite basis set in Fig. 4(a). The Hartree-Fock wavefunction does not include opposite-spin correlations, and therefore the relative wavefunction is constant at electron coalescence. The description of the gradient discontinuity in the wavefunction at coalescence improves with increasing basis set 
size. In Fig. 4(b) we repeat the exercise in the presence of the pseudopotential. The pseudopotential has zero gradient at $r=0$ so the exact wavefunction is now smooth at electron coalescence. This allows the shape of the wavefunction to be described accurately by a relatively small basis set. To quantify the change in wavefunction with basis set size, we examine in Fig. 4(c) the relative error in the wavefunction, spatially averaged within the exchange correlation hole, $k_{\mathrm{F}} r \leq \pi$, using

$$
\left\langle\frac{\Delta \psi}{\psi}\right\rangle^{2}=\frac{3 k_{\mathrm{F}}^{3}}{\pi^{3}} \int_{0}^{\pi / k_{\mathrm{F}}}\left(1-\frac{\psi_{M}(r)}{\psi_{\infty}(r)}\right)^{2} 4 \pi r^{2} \mathrm{~d} r
$$

where $\psi_{M}(r)$ is the relative wavefunction on coalescence of two opposite-spin electrons with separation $r$, calculated with CID and a basis set size $M$. The average error for the Coulomb potential falls slowly with increasing basis set size. However, a proper description of the wavefunction at coalescence requires a plane-wave basis set with a wave vector of at least $\sim 1.5 k_{\mathrm{F}}$, corresponding to a basis set size of $M=57$. Fig. 4(c) shows that here the error in the wavefunction drops markedly and the wavefunction is over ten times more accurate than that for the Coulomb interaction at the same basis set size. With large basis sets the wavefunction obtained with the pseudopotential converges more rapidly than that for the Coulomb interaction.

Now that we have shown that the pseudopotential facilitates CID calculations of the wavefunction we study the impact on evaluating the ground state energy. Both estimates tend towards the same ground state energy, confirming the accuracy of the pseudopotential. In Fig. 4(d) we show that the error in the ground state energy calculated with the Coulomb interaction scales as $1 / M[19]$ whereas with the pseudopotential it scales as $1 / M^{7 / 3}$, which is the same improvement as seen with explicitely correlated methods [19]. The pseudopotential delivers benchmark chemical accuracy of $0.017 \% E_{\mathrm{C}}=$ 0.0016 au per electron with a $\sim 50 \%$ smaller basis set and, since the computational cost of CID scales as $\mathcal{O}\left(M^{6}\right)$, this corresponds to a speed-up of a factor of $\sim 32$. Even greater computational gains could be expected at higher levels of target accuracy.

The pseudopotential has contributed to reducing the basis set size required in a CID calculation. This benefit is expected to be carried over to more accurate configuration interaction approaches, for example coupled cluster that overcomes the errors introduced into CID by unlinked diagrams [51]. Here we adopted a plane-wave basis set, however applications of configuration interaction to molecules often express the wavefunction in a coordinate basis set centered on the atoms. The pseudopotential takes a smooth polynomial form so the two-electron integrals could be evaluated efficiently as summations over the Boys function [37].
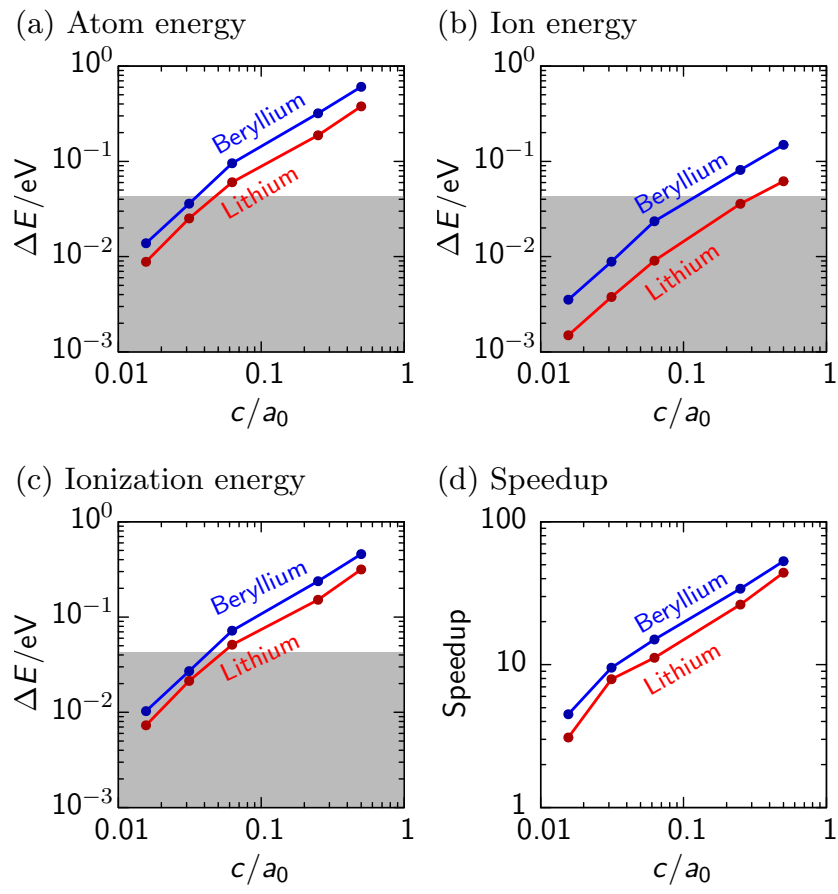

FIG. 5. (Color online) (a) Error $\Delta E$ in the total energy with cutoff radius. The red points are for the isolated $\mathrm{Li}$ atom and blue are for the Be atom. The gray shading denotes where the results attain chemical accuracy. (b) The error in the total energy of the Li (red) and Be (blue) ions. (c) The error in the ionization energy of a $\mathrm{Li}$ (red) and Be (blue) atom. (d) The speedup of the DMC calculation. The red points show the $\mathrm{Li}$ atom and blue the Be atom.

\section{LITHIUM \& BERYLLIUM ATOMS}

The HEG is arguably the most important model of interacting electrons. However, in real systems, the background charge density due to the atomic nuclei is nonuniform and so the electron density varies in space. In order to study the performance of the pseudopotential in an inhomogeneous system, we perform DMC calculations of the energy of the lithium and beryllium atoms. These atoms are simple real-life systems that could expose errors introduced by three-body scattering. Accurate reference results from analytic integration and recursion relations $[52,53]$ are also available, making these systems an ideal test bed for evaluating the performance of the electron-electron pseudopotential.

The trial wavefunction is constructed from singleparticle orbitals in a Gaussian basis set generated by an all-electron calculation performed using CRYSTAL [50]. The trial wavefunction consists of a determinant of DFT orbitals multiplied by a Jastrow correlation factor. The parameters in the Jastrow factor are optimized using a variance minimization technique [15]. The optimized $\mathrm{VMC}$ wavefunction is used as a starting point for a DMC calculation. 
We first study a solitary Li atom, containing one downspin and two up-spin electrons. We present our estimates for the ground state energy in electronvolts for ready comparison with the real-life system. In Fig. 5(a) we show the variation of the accuracy compared with the pure Coulomb interaction. The energy for the exact Coulomb system, $-203.379 \mathrm{eV}$, agrees with reference results from analytic integration and recursion relations $[52,53]$ within $0.024 \mathrm{eV}$ per atom. The error decreases as the cutoff radius is reduced. If we aim for an error of order chemical accuracy $(0.025 \mathrm{eV}$ per atom) we require $c \lesssim 0.03 a_{0}$. Fig. $5(\mathrm{~d})$ shows that relative to the calculation with the Coulomb interaction, the smoother pseudopotential reduces the local variance and therefore accelerates the calculation, with greater effect for larger cutoff radii. The pseudopotential offers a speedup by a factor of $\sim 5$ while still attaining chemical accuracy.

The results for the Be atom follow the same trend as for the $\mathrm{Li}$ atom. For the Be atom we predict a ground state energy of $-398.932 \mathrm{eV}$ per atom, again within $0.020 \mathrm{eV}$ of reference results from analytic integration and recursion relations $[52,53]$. The pseudopotential performs slightly better for the Be than the Li atom, possibly due to the increased prevalence of electron-electron relative to electron-ion interaction terms. We also determine the energy of the $\mathrm{Li}^{+}$and $\mathrm{Be}^{+}$ions in Fig. 5(b). The error is now significantly reduced due to the removal of the three-body error for $\mathrm{Li}^{+}$, and its reduction for $\mathrm{Be}^{+}$. The growth of the error in the energy estimate is similar to that for the $\mathrm{Li}$ and Be atoms. This means that in Fig. 5(c) the magnitude of the error in the ionization energy grows with cutoff radius. We attain chemical accuracy $\left(0.025 \mathrm{eV}\right.$ per atom) at $c \lesssim 0.05 a_{0}$.

For a fixed target accuracy the speedup of the pseudopotential calculation for the $\mathrm{Li}$ and $\mathrm{Be}$ atoms is smaller than for the HEG. This is because in the HEG we focused on the error per electron, whereas here we focus on the error per atom, which includes three or four electrons, therefore inflating the error. However, even if we ignore this, the electron-electron pseudopotential offers a 5-times acceleration for high accuracy work, whereas for example, for high throughput structure prediction calculations an order of magnitude less accuracy is required [54] so a pseudopotential would offer a 50-times speedup. For a molecule chemical accuracy typically relates to the energy difference between two configurations rather than total energy for which the pseudopotential is expected to be more accurate.

\section{DISCUSSION}

We have developed a pseudopotential for the repulsive Coulomb interaction. The pseudopotential delivers accurate scattering states for incident wave vectors and angular momentum channels found in an electron gas, while its smoothness accelerates computation. With the cutoff radius set to the typical electron separation the pseudopotential delivers the correct many-body physics, and within the cutoff radius two-body physics dominates where predictions for the exchange correlation hole can be corrected analytically. The cutoff radius can be reduced to zero, making the pseudopotential systematically improvable. The pseudopotential was shown to deliver chemical accuracy for the HEG and to accelerate both the DMC and CID methods by a factor of $\sim 30$. The pseudopotentials were also shown to accelerate the calculation of the isolated lithium and beryllium atom by a factor of 5 for high accuracy work, and in situations where lower accuracy is required, for example high throughput structure prediction calculations, the pseudopotentials would provide a 50 -times acceleration.

The performance and simplicity of the electronelectron pseudopotential makes it portable across manybody techniques such as VMC, DMC, truncated CI, coupled cluster theory, and Møller-Plesset theory. The formalism developed can be applied more widely in scattering problems in condensed matter to develop pseudopotentials for dipolar interactions and also the contact interactions found in atomic gases [25]. The approach can also be applied to classical physics, for example the Coulomb interaction studied here has the same force law as Newtonian gravity used in simulations of galactic dynamics [55]. Here a pseudopotential could overcome the high computational cost and correctly capture the motion of stars during close encounters.

The authors thank George Booth and Pascal Bugnion for useful discussions, and GJC acknowledges the financial support of the Royal Society and Gonville \& Caius College.

[1] W.M.C. Foulkes, L. Mitas, R.J. Needs, and G. Rajagopal, Rev. Mod. Phys. 73, 33 (2001).

[2] E.A. Hylleraas, Z. Phys. 48, 469 (1928).

[3] W. Meyer, J. Chem. Phys. 58, 1017 (1976).

[4] C. Møller and M.S. Plesset, Phys. Rev. 46, 618622 (1934).

[5] J. Č́́žek, J. Phys. Chem. 45, 4256 (1966).

[6] L. Kong, F.A. Bischoff, and E.F. Valeev, Chem. Rev. 112, 75 (2012).

[7] R.N. Hill, J. Chem. Phys. 83, 1173 (1985).

[8] W. Kutzelnigg and J.D. Morgan III, J. Chem. Phys. 96, 4484 (1992).

[9] H. Hellmann, J. Chem. Phys. 3, 61 (1935).

[10] G.B. Bachelet, D R. Hamann, and M. Schlüter, Phys. Rev. B 26, 4199 (1982).

[11] J.R. Trail and R.J. Needs, J. Chem. Phys. 122, 014112 (2005).

[12] T. Kato, Commun. Pure Appl. Math. 10, 151 (1957).

[13] R.T. Pack and W.B. Brown, J. Chem. Phys. 45, 556 (1966)

[14] S. Boys, Proc. R. Soc. London, Ser. A 258, 402 (1960). 
[15] N.D. Drummond, M.D. Towler, and R.J. Needs, Phys. Rev. B 70, 235119 (2004).

[16] W. Kutzelnigg, Theor. Chim. Acta 68, 445 (1985).

[17] W. Klopper and W. Kutzelnigg, Chem. Phys. Lett. 134, 17 (1987).

[18] W. Kutzelnigg and W. Klopper, J. Chem. Phys. 94, 1985 (1991).

[19] A. Grüneis, J.J. Shepherd, A. Alavi, D.P. Tew, and G.H. Booth, J. Chem. Phys. 139, 084112 (2013).

[20] T.M. Henderson, and R.J. Bartlett, Phys. Rev. A 70, 022512 (2004).

[21] T.M. Henderson, and R.J. Bartlett, Mol. Phys. 103, 2093 (2005).

[22] E. Clementi, IBM J. Res. Dev. 9, 2 (1965).

[23] I. Panas, Chem. Phys. Lett. 245, 171 (1995).

[24] A. Savin, Recent Developments and Applications of Modern Density Functional Theory, (Ed. J.M. Seminario) Elsevier, Amsterdam, 327 (1996).

[25] P.O. Bugnion, P. López Ríos, R.J. Needs, and G.J. Conduit, Phys. Rev. A 90, 033626 (2014).

[26] T.L. Gilbert, Rev. Mod. Phys. 35, 491 (1963).

[27] H.F. King, Theor. Chim. Acata 94, 345 (1996).

[28] M. Nooijen and R.J. Bartlett, J. Chem. Phys. 109, 8232 (1998).

[29] D. Prendergast, M. Nolan, C. Filippi, S. Fahy, and J.C. Greer, J. Chem. Phys. 115, 1626 (2001).

[30] D.R. Hamann, Phys. Rev. B 40, 2980 (1989).

[31] M. Taut, Phys. Rev. A 48, 3561 (1993).

[32] N.R. Kestner, and O. Sinanoglu, Phys. Rev. 128, 2687 (1962).

[33] P.M. Laufer, and J.B. Krieger, Phys. Rev. A 33, 1480 (1986).

[34] U. Merkt, J. Huser, and M. Wagner, Phys. Rev. B 43, 7320 (1991).

[35] T.M. Henderson, K. Runge, R.J. Bartlett, Chem. Phys. Lett. 337, 138 (2001).

[36] T.M. Henderson, K. Runge, and R.J. Bartlett, Phys. Rev. B 67, 045320 (2003).

[37] S. Reine, T. Helgaker, and R. Lindh, Wiley Interdisciplinary Reviews: Computational Molecular Science 2,
290 (2012).

[38] D.M. Ceperley and B.J. Alder, Phys. Rev. Lett. 45, 566 (1980).

[39] G. Ortiz, M. Harris, and P. Ballone, Phys. Rev. Lett. 82, 5317 (1999).

[40] F.H. Zong, C. Lin, and D.M. Ceperley, Phys. Rev. E 66, 036703 (2002).

[41] G.G. Spink, R.J. Needs and N.D. Drummond, Phys. Rev. B 88, 085121 (2013).

[42] R.J. Needs, M.D. Towler, N.D. Drummond and P. López Ríos, J. Phys.: Condensed Matter 22, 023201 (2010).

[43] P.P. Ewald, Ann. Phys. 64, 253 (1921).

[44] M.P. Tosi, in Solid State Physics, 16, edited by H. Ehrenreich and D. Turnball, Academic New York (1964).

[45] P. López Ríos, A. Ma, N.D. Drummond, M.D. Towler, and R.J. Needs, Phys. Rev. E 74, 066701 (2006).

[46] P.R.C. Kent, R.J. Needs, and G. Rajagopal, Phys. Rev. B 59, 12344 (1999).

[47] N.D. Drummond and R.J. Needs, Phys. Rev. B 72, 085124 (2005).

[48] J. Leszczynski and M.K. Shukla, Practical Aspects of Computational Chemistry, Springer (2012).

[49] R.M. Lee, G.J. Conduit, N. Nemec, P. López Ríos, and N.D. Drummond, Phys. Rev. E 83, 066706 (2011).

[50] R. Dovesi, V.R. Saunders, C. Roetti, R. Orlando, C.M. Zicovich-Wilson, F. Pascale, B. Civalleri, K. Doll, N.M. Harrison, I.J. Bush, P. DArco, and M. Llunell, CRYSTAL06 User's Manual (University of Torino, Torino, 2006).

[51] R.J. Bartlett and M. Musial, Rev. Mod. Phys. 79, 291 (2007).

[52] M. Puchalski and K. Pachucki, Phys. Rev. A 78, 052511 (2008).

[53] S.J. Chakravorty, S.R. Gwaltney, E.R. Davidson, F.A. Parpia, and C. Froese Fischer, Phys. Rev. A 47, 3649 (1993).

[54] C.J. Pickard and R.J. Needs, J. Phys.: Condens. Matter 23, 053201 (2011).

[55] J. Binney and S. Tremaine, Galactic Dynamics, Princeton University Press (2008). 